\title{
Bioconductor caAffy Quantiles Normalization
}

National Cancer Institute

\section{Source}

National Cancer Institute. Bioconductor caAffy Quantiles Normalization. NCI Thesaurus.

Code C63935.

A method for normalization based upon quantiles. This function normalizes a matrix of probe level intensities. Defined in documents available at

http://bioconductor.org/packages/1.9/bioc/html/affy.html 